\title{
Cyclosporin A curtails the progression of free light chain synthesis in the CSF of patients with multiple sclerosis
}

\author{
B N McLEAN, * P RUDGE, $†$ E J THOMPSON* \\ From the Departments of Neurochemistry*, Institute of Neurology, and the National Hospital for Nervous \\ Diseases, $\dagger$ London, $U K$
}

SUMMARY Free light chains in the cerebrospinal fluid of patients with multiple sclerosis correlate closely with disease activity. As part of a double-blind placebo controlled trial of immunosuppression using cyclosporin A in patients with multiple sclerosis, light chain analysis was performed on pre- and post-treatment CSF samples in 19 patients, nine having received cyclosporin $A$ and 10 placebo. The placebo treated patients showed continued immunological activity, as evidenced by an increase in both free kappa and lambda chains, but cyclosporin A treatment resulted in no change or an improvement in light chain response, indicative of suppression of continued activity. As cyclosporin A does not enter the CSF, this implies that systemic stimulation is at least partly responsible for continued disease activity in multiple sclerosis. Cyclosporin A may thus be effective in preventing the deterioration of patients with multiple sclerosis.

Multiple sclerosis is a demyelinating disorder of unknown aetiology, although considerable evidence exists for the importance of immunological mechanisms in the pathogenesis. Analysis of the CSF not only aids in diagnosis, but also has been found useful in assessing disease activity. In particular the presence of free light chains correlates significantly with recent demyelinating activity. ${ }^{2}$ There is no specific treatment for multiple sclerosis, so attention has focussed on the role of immunomodulating agents on established disease. So far only high dose steroids, in the form of oral prednisolone, or intravenous methylprednisolone, have been shown to have some clinical benefit on the acute relapse, ${ }^{3}$ but these do not influence the ultimate outcome, merely ameliorate the symptomatic period, and have no significant influence on immunological parameters.

A randomised prospective double-blind controlled trial using cyclosporin $A$ was thus undertaken at the National Hospital for Nervous Diseases in London to

Address for reprint requests: Dr Brendan McLean, Dept of Neurochemistry, Institute of Neurology, Queen Sq, London WCIN 3BG, UK.

Received 19 September 1988 and in revised form 3 December 1988. Accepted 12 December 1988 examine the effect of long term therapy on clinical outcome. As part of the study, immunological parameters in CSF were monitored, the results of which we present here.

\section{Materials and methods}

Patients fulfilling the international criteria for clinically definite multiple sclerosis ${ }^{4}$ were recruited and assigned randomly to receive $10 \mathrm{mg} / \mathrm{kg} /$ day cyclosporin $A$ for 2 months then $8 \mathrm{mg} / \mathrm{kg} /$ day for the next 22 months or placebo in a double blind fashion. The mean amount of cyclosporin $\mathbf{A}$ received by the subjects over the final 22 months was $7.2 \mathrm{mg} /$ $\mathrm{kg}$ /day. Blood and CSF drug levels were initially measured by radioimmunoassay (RIA) and later by high performance liquid chromatography (HPLC). Lumbar puncture was performed immediately before commencement of 2 years of therapy and again at completion. Altogether 43 patients were recruited. Of these six males and 13 females, mean age 32 and $34 \mathrm{yr}$ respectively had two lumbar CSF specimens suitable for analysis. All had established disease for 6.6 and 8.6 (range 2 to 18 ) years.

CSF samples were spun, stored at $4^{\circ} \mathrm{C}$ until analysis which was within $24 \mathrm{~h}$ of puncture. A matched serum specimen accompanied each CSF. Kappa/Lambda immunoblotting was performed as previously described.' Briefly, CSF specimens were electrophoresed on $6 \mathrm{~mm}$ rod polyacrylamide gels, a uniform amount of protein being loaded on each gel. Upon completion of the run (about $2 \mathrm{~h}$ ), 
protein was then transferred to nitrocellulose membranes by compresssion, and the membranes incubated with light chain type-specific goat anti-human antisera and finally rabbit antigoat IgG-HRP conjugate before visualising with 3-amino, 4ethylcarbazole. The resulting light chain patterns were assessed for the presence of free and bound bands. The same investigator assessed all the immunoblots. When all the patients had completed the trial, and the analyses performed, the code was broken.

\section{Results}

Measurement of cyclosporin A by RIA and HPLC gave trough levels in blood varying between 300 and $500 \mathrm{ng} / \mathrm{ml}$. In CSF all levels were less than $30 \mathrm{ng} / \mathrm{ml}$., which was the limit of detection of the assays.

Of the 43 patients recruited, 10 failed to complete the trial, either because of failure of compliance or refusal of a lumbar puncture. Eleven patients entered the trial before the technique of light chain immunoblotting was developed, and although the second CSF was analysed, insufficient sample remained from the first specimen to perform any immunoblots. A further three patients failed to have a second CSF analysed for technical reasons. This left 19 patients with two suitable CSF specimens. Nine patients received cyclosporin A (six females, three males) and 10 received placebo (seven females, three males). The table shows the analysis for each group.

The pre-treatment CSF profiles did not show any significant differences between the two groups. When total numbers of free and bound light chains were considered, the increase in the placebo group is significant ( $1 \%$ level, Wilcoxon rank sum 2 -tailed), whereas the cyclosporin group showed no significant change. A comparison of the changes in the groups shows that placebo therapy was associated with an increase in the numbers of light chains, but cyclosporin A effected no change or an improvement $(<5 \%$ probability, Fisher's exact test). Considering now free light chains only, placebo treatment resulted in a significant increase in numbers ( $1 \%$, Wilcoxon), whereas cyclosporin A resulted in no significant change. Again, comparing the two groups, placebo therapy resulted in increases in free light chain numbers, but cyclosporin A resulted in no change or fewer $(<5 \%$, Fisher's exact). There was no significant difference between and within placebo and cyclosporin A groups in bound light chains. When free light chain subtypes were examined, both Kappa and Lambda showed a significant increase with placebo therapy $(5 \%$, Wilcoxon, for both), and no change with cyclosporin A. Comparing the two groups, cyclosporin A therapy resulted in no change or a decrease in numbers and placebo an increase for both Lambda ( $<2 \%$ significance, Fisher's exact) and Kappa ( $<5 \%$ significance, Fisher's exact). In bound chains there
Table Summary of light chain analysis in 19 patients treated with either placebo or cyclosporin $A$

\begin{tabular}{|c|c|c|c|c|c|c|c|c|c|}
\hline \multirow[b]{4}{*}{ Patient } & \multicolumn{8}{|c|}{ Light chains } & \multirow[b]{4}{*}{ Treatment } \\
\hline & \multicolumn{4}{|c|}{ Pre-treatment } & \multicolumn{4}{|c|}{ Post-treatment } & \\
\hline & \multicolumn{2}{|c|}{ Free } & \multicolumn{2}{|c|}{ Bound } & \multicolumn{2}{|c|}{ Free } & \multicolumn{2}{|c|}{ Bound } & \\
\hline & $K$ & $L$ & K & $L$ & $K$ & $L$ & $K$ & $L$ & \\
\hline $5 . \mathrm{BU}$ & 0 & 0 & 0 & 0 & 0 & 0 & 0 & 1 & Cyclosporin \\
\hline 13.DU & 0 & 0 & 0 & 0 & 0 & 0 & 0 & 0 & , \\
\hline 17.FI & 0 & 0 & 0 & 0 & 0 & 0 & 0 & 0 & ", \\
\hline 18.FR & 0 & 0 & 2 & 0 & 0 & 0 & 0 & 0 & " \\
\hline 19.HA & 0 & 0 & 0 & 1 & 2 & 6 & 1 & 3 & ", \\
\hline $29.0 \mathrm{C}$ & 0 & 0 & 0 & 0 & 1 & 0 & 0 & 0 & ", \\
\hline 34.RE & 0 & 0 & 0 & 1 & 0 & 8 & 0 & 2 & ", \\
\hline 38.TH & 2 & 6 & 2 & 2 & 2 & $\begin{array}{l}0 \\
5\end{array}$ & 2 & 2 & ", \\
\hline 40.WA & 0 & 0 & 0 & 0 & 0 & 0 & 0 & 0 & ," \\
\hline 3. BE & 0 & 0 & 1 & 2 & 6 & 7 & 3 & 0 & Placebo \\
\hline 4. BO & 0 & 0 & 0 & 0 & 0 & 0 & 0 & 0 & , \\
\hline 14.ED & 0 & 0 & 1 & 0 & 8 & 2 & 3 & 0 & $"$ \\
\hline $20 . \mathrm{HI}$ & 0 & 1 & 0 & 0 & 3 & 7 & 2 & 3 & ," \\
\hline 26.LA & 0 & 0 & 1 & 1 & 3 & 7 & 0 & 1 & $"$ \\
\hline 27.MA & 0 & 1 & 1 & 0 & 3 & 3 & 1 & $i$ & $"$ \\
\hline 30.PE & 0 & 2 & 0 & 2 & 0 & 8 & 0 & 2 & ”, \\
\hline 36.RU & 2 & 5 & 1 & 1 & 0 & 2 & 3 & 6 & ", \\
\hline 39.WA & 0 & 0 & 0 & $i$ & 1 & 4 & 0 & 1 & ”, \\
\hline 42.WY & 0 & 3 & 2 & 3 & 6 & 10 & 3 & 1 & ", \\
\hline
\end{tabular}

$\mathrm{K}=\mathrm{Kappa}, \mathrm{L}=\mathrm{Lambda}$, numbers refer to bands seen on blots of electrophoresed CSF.

were no significant changes in either subtype on any test for both groups.

\section{Discussion}

Free light chains in the CSF of patients with neurological disease originate from intrathecal synthesis and not degradation of IgG. ${ }^{67}$ They reflect disease activity, but are not disease specific, occurring in a variety of neurological disorders, including multiple sclerosis. ${ }^{8}$ Increased numbers of free light chains are associated with recent antigenic stimulation in multiple sclerosis, but not apparently with clinical disability.' The reason for this elevation is unclear, but probably reflects increased turnover in stimulated Blymphocytes. In our study the changes in light chain numbers were entirely due to the free fraction, and it was in the placebo treated group only that significant increases were seen. Cyclosporin A treated patients had no change, or a decrease in free light chain numbers. Since free light chains indicate active disease, this suggests that in the placebo treated group there was continuing demyelination and that cyclosporin $\mathbf{A}$ caused a decrease in activity. Clinical studies on the two groups also suggests that cyclosporin $A$ appeared to halt deterioration (to be published). It has been established in this series and others, however, that cyclosporin A does not enter the CSF in therapeutic or even detectable quantities. This implies that the mechanism of action of cyclosporin is on the systemic 
immune system and supports the concept that it is systemic immune activation that triggers CNS demyelination or contributes to its maintenance. Other workers have found that cyclosporin A suppresses the continued and progressive immune activation in the serum of multiple sclerosis patients, as evidenced by T-cell changes. ${ }^{9}$ Subtype analysis of the light chains demonstrates that there are significant increases in both Kappa and Lambda with disease progression, and that cyclosporin $\mathrm{A}$ affects both similarly. This does not support the findings of some workers, that there is a dominant Kappa response in multiple sclerosis, ${ }^{6}$ but agrees with others. ${ }^{8}$

This study shows that cyclosporin $A$ is effective in reducing continued immune activity in patients with multiple sclerosis, and thus may arrest the clinical deterioration.

\section{References}

1 Vakaet A, Thompson EJ. Free light chains in the cerebrospinal fluid: an indicator of recent immunological stimulation. J Neurol Neurosurg Psychiatry 1985;48:995-8.

2 Bracco F, Gallo P, Menna R, Battistin L, Tavolato B.
Free light chains in the CSF in multiple sclerosis. $J$ Neurol 1987;234:303-7.

3 Compston DAS, Milligan NM, Hughes PJ, et al. A double-blind controlled trial of high dose methylprednisolone in patients with multiple sclerosis: 2 . Laboratory results. J Neurol Neurosurg Psychiatry 1987;50:517-22.

4 Poser CM, Paty DW, Scheinberg L, et al. New diagnostic criteria for multiple sclerosis: guidelines for research protocols. Ann Neurol 1983;13:227-31.

5 Thompson EJ, Keir G. Improved detection of oligoclonal and bence-jones proteins by $\mathrm{k} / \mathrm{l}$ immunoblotting . Clin Chem Acta 1984;143:329-35.

6 Rudick RA, Pallant A, Bidlack JM, Herndon RM. Free kappa light chains in multiple sclerosis spinal fluid. Ann Neurol 1986;20:63-9.

7 Rudick RA, Peter DR, Bidlack JM, Knutson DW. Multiple sclerosis: free light chains in cerebrospinal fluid. Neurology 1985;35:1443-9.

8 Fagnart OC, Sindic CJM, Laterre C. Free kappa and lambda light chain levels in the cerebrospinal fluid of patients with multiple sclerosis and other neurological diseases. J Neuroimmunol 1988;19:119-32.

9 Kerman RH, Wolinsky JS, Nath A, Sears ES. Serial immune evaluation of cyclosporin- and placebotreated multiple sclerosis patients. $J$ Neuroimmunol 1988;18:325-31. 